\title{
ESTIMATIVA DE ESTÁDIOS NINFAIS DO PSILÍDEO-DE-CONCHA EM FUNÇÃO DOS TAMANHOS DAS CONCHAS
}

\author{
Aline Stivanelli - bolsista Embrapa/CNPq-ITIa, PUCCAMP Campinas/SP, aline.stivanelli@yahoo.com.br \\ Maria Conceição Peres Young Pessoa - Pesquisador A/Embrapa Meio Ambiente, Jaguariúna/SP, \\ young@cnpma.embrapa.br; \\ Luiz Alexandre Nogueira de Sá - Pesquisador A- LQC/Embrapa Meio Ambiente, Jaguariúna/SP, \\ lans@cnpma.embra.br e \\ Jodir Pereira da Silva - Prof. Dr. Oceanografia - CCV/ PUCAMP, Campinas/SP - prof.jodir@gmail.com
}

\section{RESUMO}

O psilídeo-de-concha, Glycaspis brimblecombei, é considerado praga quarentenária de elevada importância econômica para o setor florestal no Brasil pela preferência a Eucaliptus camaldulensis - espécie mais plantada no país. A insuficiência de métodos químicos, aceitáveis pelas empresas certificadas internacionalmente, para o controle da praga vem tornando a estratégia de controle biológico pelo parasitóide Psyllaephagus bliteus a opção mais apropriada no momento. Por esta razão, a disponibilidade de métodos que ajudem a detectar fases de desenvolvimento da praga mais apropriadas ao parasitismo é de grande importância tanto para a identificação precisa do seu ciclo de vida quanto à proposição de estratégias de seu controle biológico. $\mathrm{O}$ objetivo deste trabalho foi identificar faixas de tamanhos de concha de G. brimblecombei, de modo a utilizá-las como padrão de referencia para um método inédito de estimativas de estádios ninfais de desenvolvimento da praga em função do seu respectivo tamanho de concha amostrado. Essas faixas (ou classes de tamanhos) foram obtidas através de minucioso trabalho entomológico realizado em laboratório, onde os diâmetros das conchas e seus respectivos insetos foram medidos com auxílio de lupa e avaliados quanto aos estádios de desenvolvimento. Posteriormente, foram realizadas análises estatísticas e de tendências. As seguintes classes de tamanhos de concha foram determinadas como resultado: Ninfa $1-$ de $0,7 \mathrm{~mm}$ a $0,9 \mathrm{~mm}$; Ninfa 2 - de $0,8 \mathrm{~mm}$ a $1,1 \mathrm{~mm}$; Ninfa 3 - de $1,1 \mathrm{~mm}$ a 1,7 $\mathrm{mm}$; Ninfa 4 - de 1,6 $\mathrm{mm}$ a 2,2 mm;e Ninfa 5 - de $2,2 \mathrm{~mm}$ a $2,8 \mathrm{~mm}$.

Palavras-chave: Psyllidae; Encyrtidae; controle biológico; instar; floresta.

\section{ESTIMATES OF RED-GUM LERP PSYLLID NYMPHAL INSTARS BASED ON THEIR SHELL SIZES}

\section{ABSTRACT}

The red-gum lerp psyllid is considered a pest in Brazil, whose preference for Eucalyptus camaldulensis - a species of high economic importance- has caused great concern to the national forest sector. The lack of pesticides acceptable by internationally certified companies to control the pest turns the strategy of biological control by the parasitoid Psyllaephagus bliteus the most appropriate option. Thus, the availability of methods which could help to identify the plague developmental phases preferable for the parasitoid, have great importance both for the correct description of its life cycle, as well as, for proposals applied to its biological control strategies. The main objective of the present work was to identify the shell size ranges of Glycaspis brimblecombei in order to use them as a reference pattern for a new method, which intends to estimate the nymphal stages based on their respective shell size sampled. These ranges (or size classes) were reached using a careful entomologic method conducted at laboratory conditions, where the shell diameters and insects were both measured with a 
magnifying glass and later evaluated in order to identify their nymphal instar. Afterward, statistic and tendency analysis were performed. The following shell size classes were established as result: Nymph 1 - 0.8 mm; Nymph 2 - from 0.8 $\mathrm{mm}$ to $1.1 \mathrm{~mm}$; Nymph 3 - from $1.1 \mathrm{~mm}$ to 1.7 $\mathrm{mm}$; Nymph 4 - from $1.6 \mathrm{~mm}$ to $2.2 \mathrm{~mm}$; and Nymph 5 - from $2.2 \mathrm{~mm}$ to $2.8 \mathrm{~mm}$.

Key-words: Psyllidae; Encyrtidae; biological control; instars; forest.

\section{INTRODUÇÃO}

O Brasil possui a maior área do mundo de plantação de eucalipto (Mct, 2005) que vem sofrendo relevantes perdas com a introdução de pragas exóticas no país (Firmino, 2004). Com elevada importância econômica, o eucalipto vem sendo utilizado, principalmente, na produção de celulose, papel, chapa de fibra, carvão vegetal (no abastecimento às siderúrgicas), lenha, serrados, compensados e lâminas, além de painéis reconstituídos, como aglomerados e MDF. Ressaltando ainda sua importância social, em 2005 eram gerados quatro milhões de empregos diretos ou indiretos a partir das plantações de eucalipto em conjunto com outras culturas do setor florestal brasileiro (Mct, 2005; Aracruz, 2008; Carvalho, 2006).

Dentro do estado de São Paulo verifica-se a produção em larga escala em praticamente toda a sua extensão (Cati, 2008). Rapassi et al. (2008) acrescenta ainda a existência de potencial de expansão de cultivo do estado para a região oeste. Esse potencial de produção de eucalipto, como nas demais regiões do país, faz com que a cultura sofresse influências climáticas e a proximidade taxonômica com diversas espécies brasileiras favorecendo a adaptação de muitos insetos. Os extensos plantios, homogêneos e contínuos, distribuídos por todo o país forneceram grande quantidade de alimentos a estes insetos que, aliada à baixa diversidade interferiu no equilíbrio ecológico dessas populações possibilitando seu aumento descontrolado, tornando-os pragas (Rapassi et al., 2008).
Glycaspis brimblecombei (Hemiptera: Psyllidae), conhecido como psilídeo-de-concha devido à formação de pequenos cones brancos semelhantes às conchas, se desenvolve nessa estrutura até a fase adulta (Sá \& Wilcken, 2004). Nativa da Austrália, a praga preferencialmente ataca Eucaliptus camaldulensis (Brennan et al., 1998). Segundo Hoddle (2004) possui hábito sugador causando enrolamento e deformação do limbo foliar, secamento dos ponteiros, entre outros, podendo levar o vegetal à morte. É um inseto diminuto, medindo no máximo $4 \mathrm{~mm}$ de comprimento, levando de 20 a 45 dias para realizar o ciclo completo de desenvolvimento. Apresenta cinco estádios ninfais diferenciados, identificados a partir da quantidade de segmentos presentes em suas antenas (Sá \& Wilcken, 2004). No controle da praga, inseticidas de contato ou sistêmicos não tem se mostrado eficientes, além de apresentarem potencial para gerar impacto ambiental negativo e custo elevado. Desse modo o parasitóide Psyllaephagus bliteus (Hymenoptera: Encyrtidae) vêm sendo apontado como o método de controle mais apropriado do psilídeo-de-concha. Suas fêmeas colocam ovos no abdome das ninfas de G. brimblecombei, preferencialmente em $3^{\circ}$ e $4^{\circ}$ estádios ninfais, levando-as à morte (Sá \& Wilcken, 2004). Por esta razão, a determinação de lacunas sobre estes estádios de desenvolvimento é fundamental para a correta proposição de estratégias de controle que façam uso do parasitóide (Pessoa etal., 2007). As particularidades das espécies citadas tornam a identificação desses estádios um trabalho minucioso e de difícil avaliação. Desse modo, a proposição de métodos que auxiliem à melhoria da qualidade na identificação e quantificação desses estádios preferenciais é de importância fundamental para a definição mais fidedigna de estádios do ciclo de desenvolvimento da praga e de estratégias mais apropriadas ao controle biológico pelo parasitóide.

Este trabalho teve por objetivo principal identificar faixas de tamanhos de concha de G. brimblecombei, de modo a utilizálas como padrão de referencia para um método 
inédito de estimativas de estádios ninfais de desenvolvimento da praga em função do seu respectivo tamanho de concha amostrado.

\section{MATERIAL E MÉTODOS}

O presente trabalho foi realizado no Laboratório de Quarentena "Costa Lima", da Embrapa Meio Ambiente, Jaguariúna/SP, no âmbito do Projeto "Desenvolvimento de métodos e aplicativos para sistemas quarentenários em apoio à defesa agropecuária nas culturas de citros, cana-de-açúcar, eucalipto e flores/plantas ornamentais no estado de São Paulo" (Edital CNPQ/MAPA No.64/2008).

Utilizou-se uma gaiola de criação, identificada pelo código GC08, contendo em seu interior 42 tubetes plantados E. camaldulensis e disposta em sala de criação de insetos mantida a temperatura a $25,4 \pm 0,2{ }^{\circ} \mathrm{C}$, UR $50,3 \pm 4,7 \%$ e fotofase de $12 \mathrm{~h}$. Nela foram introduzidos 40 fêmeas e 60 machos adultos do psilídeode-concha. Os tubetes foram diariamente monitorados e regados pelo período de 65 dias, iniciado após o aparecimento dos primeiros ovos. Fazendo uso de uma gratícula de Porton NG2 (calibrada com a gratícula da platina) acoplada a uma ocular de microscópio adaptado à uma lupa modelo WILD HEERBRUGG Plan 1x 50 vezes, pinça e pincel de pelo de marta, foram feitas as medidas dos tamanhos de conchas e ninfas e identificados os respectivos estádios ninfais. As conchas foram levantadas sem danificá-las, uma a uma, a fim de se identificar, quantificar seu tamanho (diâmetro em $\mathrm{mm}$ ) e resguardar a integridade da ninfa em seu interior mantendo-a viva. Ambas foram fotografadas em Câmera Digital Sony Cybershot DSC-S730 (7,2 Megapixels).

Na identificação do estádio ninfal levouse em consideração: a) coloração e rigidez da concha amostrada (que variaram devido ao processo de construção em que se encontravam - conchas em formação ou recém-formadas são mais transparentes ou leitosas e mais maleáveis, em contraponto, àquelas que estão formadas há mais tempo, que já são bem esbranquiçadas e rígidas); b) ninfas em estádio intermediário (que foram classificadas como sendo do estádio seguinte. Por exemplo, sabendo-se que uma ninfa de $2^{\circ}$ estádio possui três segmentos de antena e uma de $3^{\circ}$ possui cinco segmentos, ninfas com quatro segmentos de antena foram classificadas como sendo de $3^{\circ}$ estádio); e c) conchas encontradas vazias ou com ninfas mortas foram desconsideradas. Essa parte biológica de medição de conchas e ninfas, bem como análise entomológica de estádio das mesmas gerou maior dificuldade do que o inicialmente previsto devido ao tamanho diminuto e delicadeza do insetopraga em fase imatura de desenvolvimento e de sua concha, sendo que em toda realização do experimento priorizou-se manter a integridade das ninfas. Houve grande exigência também no que se diz respeito ao avaliador dos tamanhos, sendo que todas as medidas se tratavam de milímetros e o esforço repetitivo gerou fadiga ocular, especialmente para os $3^{\circ}$ e $4^{\circ}$ ínstares preferidos pelo parasitóide $P$. bliteus. Talvez essa seja a razão da ausência de determinações de tempos de duração e viabilidade das fases ninfais da praga em artigos já disponibilizados na literatura nacional e internacional.

Os dados foram, posteriormente, organizados em planilha Mycrosoft Excelle avaliados estatisticamente, separadamente por estádios ninfais identificados. Assim, foram calculados os tamanhos médios das ninfas (em $\mathrm{mm}$ ) para cada valor de tamanho de concha (em mm). Gráficos de tamanho de concha $\mathrm{X}$ tamanho médio da ninfa foram elaborados para cada estádio ninfal avaliado. Posteriormente foi utilizado o método dos mínimos quadrados (Dorn, 1981) para a realização de análise de regressão. A partir desses resultados, estabeleceu-se um padrão de referência para uso no método de identificação de estádios ninfais por tamanhos das conchas (diâmetros em mm).

\section{RESULTADOS E DISCUSSÕES}

Durante os 65 dias de realização do experimento foram fotografados e medidos um total 
de 370 ninfas, e respectivos tamanhos de conchas (diâmetro em mm), sendo que: 90 de $1^{\circ}$ estádio, 80 de $2^{\circ}, 70$ de $3^{\circ}, 70$ de $4^{\circ}$ e 60 de $5^{\circ}$ estádio. Posteriormente, estes dados foram tratados e analisados estatisticamente, inicialmente pelo teste de normalidade de Lilliefors (Campos, 1979) considerando $p<0,01$, para verificação de normalidade das populações observadas. Posteriormente, utilizou-se o teste de comparação de médias de Tukey, que comprovou que as ninfas do psilídeo apresentaram diferenças significativas quando avaliados os tamanho de concha e tamanho de ninfa de um estádio para o outro. Posteriormente foram feitas análises de tendências sendo possível estabelecer curvas de regressão polinomial pelo método dos mínimos quadrados (Triola, 1999), cujos resultados médios estão apresentados na Figura 1.

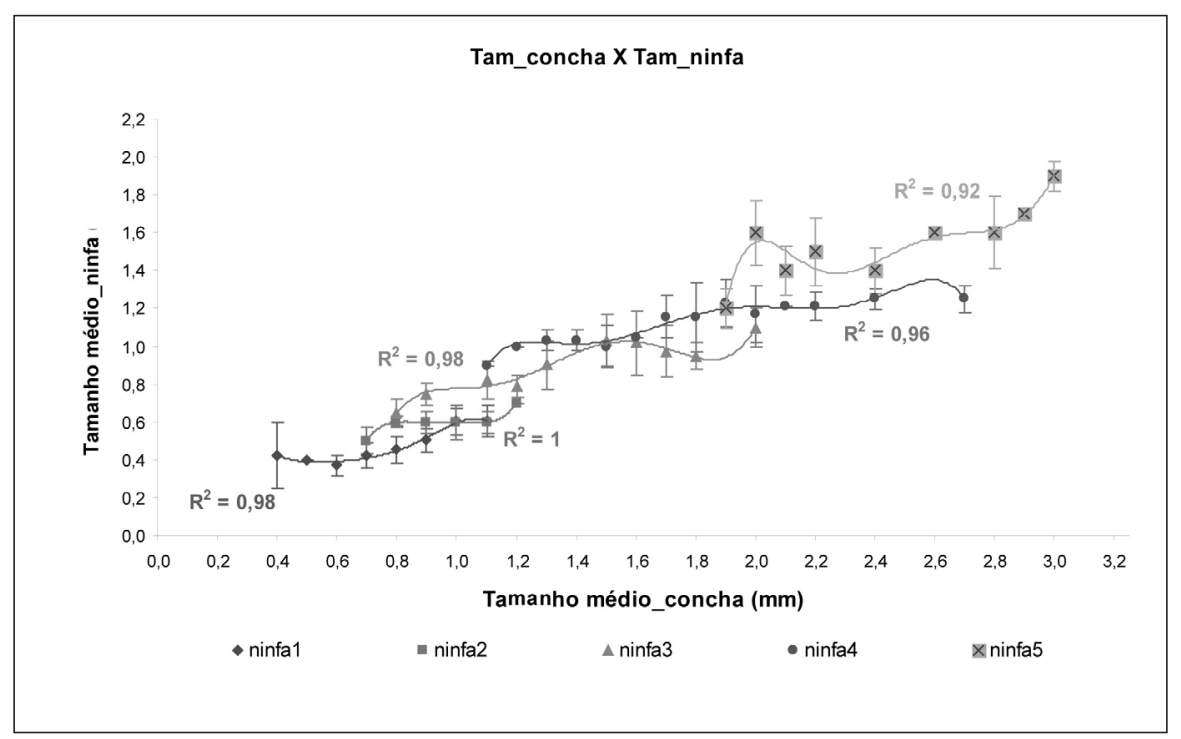

FIGURA 1. Tamanho de concha X Tamanhos médios de estádios ninfais de G. brimblecombei em gaiola de criação.

As equações ajustadas por regressão polinomial, e repectivos coeficiente de determinação $\left(\mathrm{R}^{2}\right)$, são apresentadas a seguir:

$$
\mathrm{y}_{\text {Tam_nin1 }}=-23,406 \times 5+82,328 \times 4=
$$

$113,59 \times 3^{-}+77,874 \times 2-26,635 x+4,0229$ $\mathrm{R}^{2}=0,98$

$$
\mathrm{y}_{\text {Tam_nin2 }}=166,67 \times 5-791,67 \times 4+
$$
$1500 x 3-1417,1 \times 2+667,48 x-124,8$ $\mathrm{R}^{2}=1,00$

$$
\mathrm{y}_{\text {Tam_nin3 }}=-2,2587 \times 6+29,168 \times 5-134,53 \times 4
$$

$+301,18 \times 3-354,28 x 2+210,88 x-49,384$ $\mathrm{R}^{2}=0,98$

$$
\mathrm{y}_{\text {Tam_nin } 4}=-5,1264 \times 6+58,107 \times 5-269,7 \times 4
$$

$+655,54 \mathrm{x} 3-879,46 \mathrm{x} 2+617,41 \mathrm{x}-176,28$

$\mathrm{R}^{2}=0,96$$$
\mathrm{y}_{\text {Tam nin5 }}=-28,648 \times 6+447,27 \times 5-
$$ $2889,7 \times 4+9889 x 3-18906 \times 2+19145 x-8020,5$ $\mathrm{R}^{2}=0,92$.

Após análise estatística dos dados médios obtidos, observaram-se os intervalos correspondentes aos tamanhos da concha, máximo e mínimo, para cada estádio ninfal, apresentados a seguir: Ninfa 1 - de $0,7 \mathrm{~mm}$ a $0,9 \mathrm{~mm}$; Ninfa 2 - de $0,8 \mathrm{~mm}$ a $1,1 \mathrm{~mm}$; Ninfa 3 - de 1,1 mm a 1,7 mm; Ninfa 4 - de 1,6 mm a 2,2 $\mathrm{mm}$; e Ninfa 5 - de 2,2 $\mathrm{mm}$ a 2,8 $\mathrm{mm}$.

Observa-se pelos resultados apresentados que, à medida que as ninfas se desenvolvem e passam de um estádio para outro, os tamanhos de suas conchas podem se sobrepor nos 
primeiros dias de observação de $2^{\circ}, 3^{\circ}, 4^{\circ}$ e $5^{\circ}$ estádios. Esta observação é biologicamente explicada em função das características individuais de desenvolvimento de cada ninfa e em função do exato momento de observação e medição das mesmas. Partindo-se do princípio de que, se uma ninfa foi observada em seu primeiro dia em determinado estádio e outra, de mesmo estádio, foi observada no último dia do mesmo, biologicamente, a primeira ninfa observada tende a ser menor que a segunda e, vice-versa.

Além dos resultados supra citados, também foi possível avaliar os tamanhos médios das ninfas (longitudinal em $\mathrm{mm}$ ) em cada estádio ninfal. Assim, observou-se que: ninfas de $1^{\circ}$ estádio medem de $0,4 \mathrm{~mm}$ à $0,6 \mathrm{~mm} ; 2^{\circ}$ estádio de $0,5 \mathrm{~mm}$ à $0,7 \mathrm{~mm} ; 3^{\circ}$ estádio de $0,8 \mathrm{~mm}$ à $1,0 \mathrm{~mm} ; 4^{\circ}$ estádio de $1,1 \mathrm{~mm}$ à $1,3 \mathrm{~mm}$ e de $5^{\circ}$ estádio de $1,4 \mathrm{~mm}$ à $1,8 \mathrm{~mm}$. A mesma explicação biológica atribuída aos tamanhos de concha pode ser aplicada aos tamanhos de ninfa.

\section{CONCLUSÃO}

Através desse estudo foi possível identificar o tamanho médio das conchas (diâmetros em $\mathrm{mm}$ ) de Glycaspis brimblecombei associados aos respectivos estádios ninfais. O trabalho também viabilizou as estimativas de tamanhos médios das ninfas (longitudinal em mm) de cada estádio ninfal de G. brimblecombei, além de estimativas dos tamanhos de ninfas em função do tamanho da concha amostrada.

As informações obtidas são entomologicamente inéditas e de fundamental importância para agilizar a correta determinação do ciclo de desenvolvimento de G. brimblecombei, no que se refere à viabilidade e duração dos estádios ninfais. Também fornece métodos e subsídios para orientar a estimativa de ninfas preferenciais ao parasitóide Psyllaephagus bliteus, em ambientes que estejam sendo prejudicados pela praga G. brimblecombei, a fim de realizar com melhor eficácia sua liberação para fins de controle biológico da praga.

\section{REFERÊNCIAS BIBLIOGRÁFICAS}

ARACRUZ. Eucalipto e meio ambiente. 2008. Disponível em: <www.aracruz.com.br $>$. Acesso em: 17/09/2009.

BRENNAN，E. B.; GILL，R.J.; HRUSA, G.F.; WEINBAUM, S.A. First Record of Glycaspis brimblecombei (Moore) (Hemyptera: Psyllidae) in North America: initial observations of potentially serious pest of eucalyptus in California. Pan-Pacific Entomologist, 1998, v.75, n.1, p.55-57.

CAMPOS, H. Estatística experimental nãoparamétrica. Piracicaba: USP/ESALQ, 1979. 343p.

CARVALHO, A.D.F. Histórico do melhoramento genético de eucalipto no Brasil. Piracicaba/SP, 2006. Disponível em: $<$ http://www.genetica.esalq.usp.br/semina. php>. Acesso em: 17 set 2009.

DORN, W. S. Cálculo numérico com estudos de casos em Fortran IV. Rio de Janeiro: Campus; São Paulo: Ed. da Universidade de São Paulo, 1981. 568p.

FIRMINO, D. C. Biologia do psilídeo-deconcha Glycaspis brimblecombei Moore (Hemyptera: Psyllidae) em diferentes espécies de eucalipto e em diferentes temperaturas. Botucatu, 2004. 49 p.

HODDLE, M., DAHLSTEN, D. , KABASHIMA, J. Biology and Management of the Redgum Lerp Psyllid, Glycaspis brimblecombei. California. Public works, 2004. 3 p. Disponível em:<http://www.ci.manhattan-beach.ca.ua/ pubworks/lerp/kabashim.html>. Acesso em: 21 mar. 2009.

MEDRADO, M. J. S. Cultivo do eucalipto Importância socioeconômica e ambiental. 
Embrapa Florestas. Sistema de produção 4. ISSN 1678-8281. Agosto, 2003. 1p. Disponível em: $<$ http://sistemasdeproducao.cnptia.embrapa.br/ FontesHTML/Eucalipto/CultivodoEucalipto/01 Importancia_economica.html>. Acesso em: $1 \overline{6}$ set. 2009.

\section{MINISTÉRIO DA CIÊNCIA E TECNOLOGIA.} Especial Projeto Genolyptus. Disponível em: <http://www.mct.gov.br/especial/genolyptus4html>. Acesso em: 27 fev. 2009.

PESSOA, M.C.P.Y; SÁ, L.A.N.; KODAIRA, J.Y.; WILCKEN, C.F.; ALMEIDA, G.R. Avaliação de estratégias de criação laboratorial visando o controle biológico do psilídeo-de-concha, Glycaspis brimblecombei(Hemíptera:Psyllidae) porparasitismo de Psyllaephagus bliteus (Hymenoptera: Encyrtidae) por simulação de sistemas. Simpósio de Entomologia,
Anais... 2007, Campina Grande/PB, 135 p.

RAPASSI, R.M. A. et al. Cultura do eucalipto na região de Suzanápolis Estado de São Paulo: análise econômica. Informações Econômicas, São Paulo, v.38, n.4, p.7-13, 2008.

SÁ, L.A.N.; WILCKEN, C.F. Nova Praga Exótica no Ecossistema Florestal. Jaguariúna. Comunicado técnico. 2004. 3 p.

SÃO PAULO (Estado). Secretaria de Agricultura e Abastecimento. Coordenadoria de Assistência Técnica Integral. Instituto de Economia Agrícola. Levantamento censitário de unidades de produção agrícola do Estado de = São Paulo - LUPA 2007/2008. São Paulo: SAA/CATI/IEA, 2008. Disponível em: <http://www.cati.sp.gov. br/projetolupa >. Acesso em: 16 ago 2009. 